\title{
Particle Removal in Ultrasonic Water Flow Cleaning: Role of Cavitation Bubbles as Cleaning Agents
}

\author{
Keita Ando ${ }^{1, a^{*}}$, Mao Sugawara ${ }^{1, b}$, Riria Sakota ${ }^{1, c}$, \\ Tomoatsu Ishibashi ${ }^{2, \mathrm{~d}}$, Hisanori Matsuo ${ }^{2, \mathrm{e}}$, and Katsuhide Watanabe ${ }^{2, \mathrm{f}}$ \\ ${ }^{1}$ Keio University, 3-14-1 Hiyoshi, Kohoku-ku, Yokohama, Kanagawa 223-8522, Japan \\ ${ }^{2}$ Ebara Corporation, 4-2-1 Honfujisawa, Fujisawa, Kanagawa 251-8502, Japan \\ kando@mech.keio.ac.jp
}

Keywords: Ultrasonic water flow cleaning, cavitation bubbles, particle removal, visualization

\begin{abstract}
Visualization experiments are performed to examine the role of acoustic cavitation bubbles that appear in $0.43-\mathrm{MHz}$ ultrasonic water flow spreading over glass surfaces in the context of physical cleaning. The cleaning performance is evaluated using glass samples on which small silica particles are spin-coated. The visualization suggests that acoustic cavitation bubbles play a major role in particle removal as in the case of conventional cleaning with ultrasonic cleaning baths.
\end{abstract}

\section{Introduction}

In post-CMP cleaning in semiconductor industry, there is a need to remove very small contaminant particles from silicon wafers. One of the promising candidates for effective post-CMP cleaning is based on ultrasound-superposed water jets. Here, we call this technique ultrasonic water flow cleaning. When it comes to achieving particle removal, it is essential to create wall shear stress that is strong enough to defeat particle adhesion such as van der Waals force [1]. Recent studies on conventional cleaning with ultrasonic cleaning baths $[2,3,4]$ suggest that instantaneous liquid flow acceleration induced by ultrasound propagation (without cavitation) plays a minor role in ultrasonic cleaning but near-wall liquid flow promoted by dynamics of acoustic cavitation bubbles at or near cleaning targets plays an essential role as main cleaning agents. We speculate that acoustic bubble dynamics come into play also in the case of ultrasonic water flow cleaning.

In this work, we aim to experimentally clarify the role of acoustic cavitation bubbles in ultrasonic water flow cleaning. Cleaning tests with ultrasound-superposed water flow $(0.43 \mathrm{MHz})$ are performed using particle-attached glass samples. Cavitation events above the glass surface over which the water flow spreads radially are visualized, which allows for exploring the relation between cavitation bubbles and particle removal.

\section{Experimental Method}

The experimental setup of surface cleaning with ultrasonic water flow is depicted in Fig. 1(a). Airsaturated water at room temperature is pumped to an ultrasonic water flow cleaner (QUAVA Spot 27220 , KAIJO; $20 \mathrm{~W}$ or $10 \mathrm{~W}$ ) and $0.43 \mathrm{MHz}$ ultrasonic water flow at volume flow $1.0 \mathrm{~L} / \mathrm{min}$ is generated. The ultrasonic water jet (along the downward direction) impinges at distance $20 \mathrm{~mm}$ from the nozzle exit ( $5 \mathrm{~mm}$ in diameter) upon a circular glass plate $(30 \mathrm{~mm}$ in diameter) as a cleaning target. The glass surface is spin-coated with silica particles $(0.5 \mu \mathrm{m}$ in diameter $)$ as contamination. The surface cleaning process is visualized with a high-speed camera (VW-9000, KEYENCE) and LED lighting (SLG-150V-CW-MN, REVOX) from the bottom; see Fig. 1(b). 
(a)

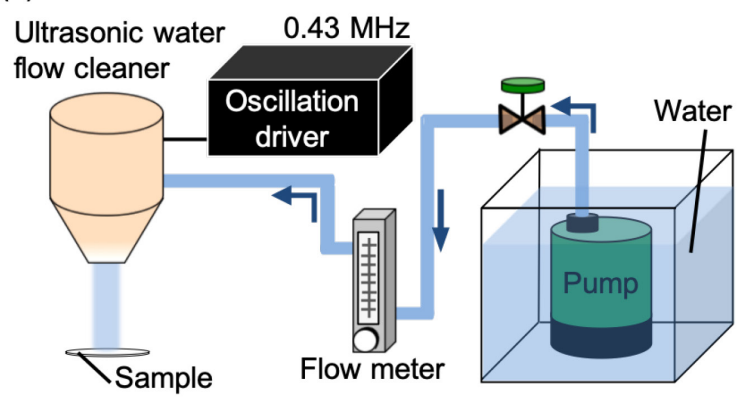

(b)

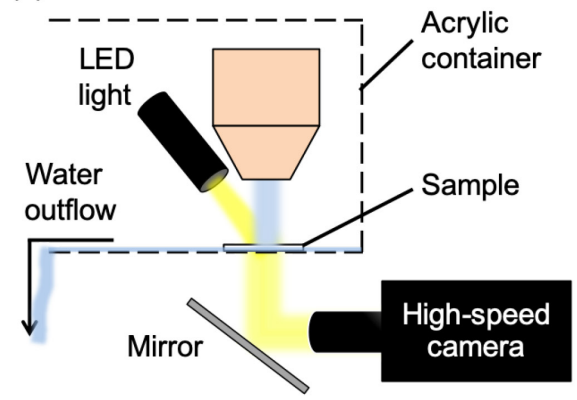

Fig. 1. Experimental setup: (a) Ultrasonic water flow cleaning of a circular glass sample spin-coated with silica particles; (b) Visualization of the glass surface during the cleaning.

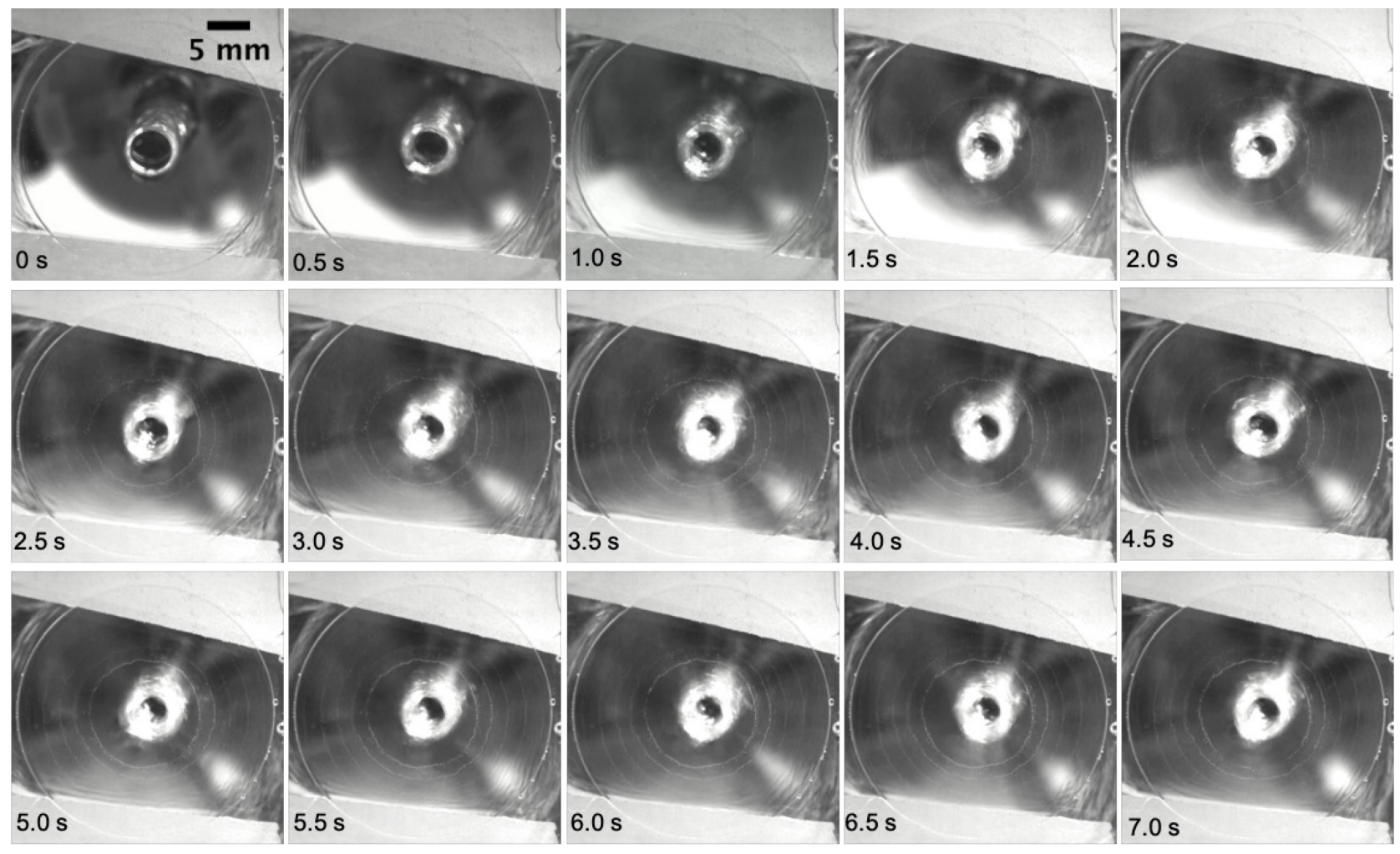

Fig. 2. Visualization (frame rate: $30 \mathrm{fps}$, exposure time $16.7 \mathrm{~ms}$, spatial resolution $62.5 \mu \mathrm{m} / \mathrm{pixel}$ ) of the glass surface (with which the higher-intensity $(20 \mathrm{~W})$ ultrasonic water flow collides) at different times after the ultrasound irradiation starts. Equally-spaced circular patterns of cavitation bubbles trapped at the surface appear at later times.

\section{Results and Discussion}

Visualization of the evolution of the glass surface during the cleaning test with the higher-intensity $(20 \mathrm{~W})$ ultrasonic water flow is presented in Fig. 2. It turns out that the population of acoustically generated cavitation bubbles above the glass surface increases over seconds and there appear equallyspaced circular patterns of trapped bubbles that are centered at the jet collision. The separation between the circular stripes is found to coincide with one half of the wavelength of $0.43 \mathrm{MHz}$ ultrasound. This suggests that a standing-wave-like pressure field arises from a superposition of the incident wave and the reflected wave propagating back from the edge of the liquid film, which is located near the glass sample's edge, and acoustic bubbles are trapped, via the so-called primary Bjerknes force [5], at node and antinode of the pressure field. The close-up view of the evolution of such cavitation bubbles is presented in Fig. 3. This also shows that the bubble population increases on the order of seconds. The radius of these bubbles that grow as a result of rectified mass diffusion 
[6] is some microns and thus close to the Minnaert resonant radius [3]. The stripe-shaped bubble cluster that appears at later times will correspond to one of the circular patterns of acoustically trapped bubbles in Fig. 2.

From the view point of cleaning, we next examine particle removal by the ultrasonic water flow. In Fig. 4, we compare the cavitation bubble structure (in Fig. 2) to the particle removal pattern (after the 30-s sonication) that can be identified from light scattering. It follows that the nonuniformity in the particle removal corresponds to the circular patterns of the trapped cavitation bubbles. In the neighborhood of oscillating bubbles, strong wall shear stress is expected to appear and thus contributes to particle removal [7]. However, fluid-dynamic force from the water flow without mixing effect of oscillating bubbles is too weak to achieve particle removal. This fact can also be confirmed in the case of the lower-intensity $(10 \mathrm{~W})$ ultrasonic water flow; see figure 5 . In this case, the bubble population is much reduced in comparison to the higher-intensity $(20 \mathrm{~W})$ case, giving rise to lower cleaning performance.

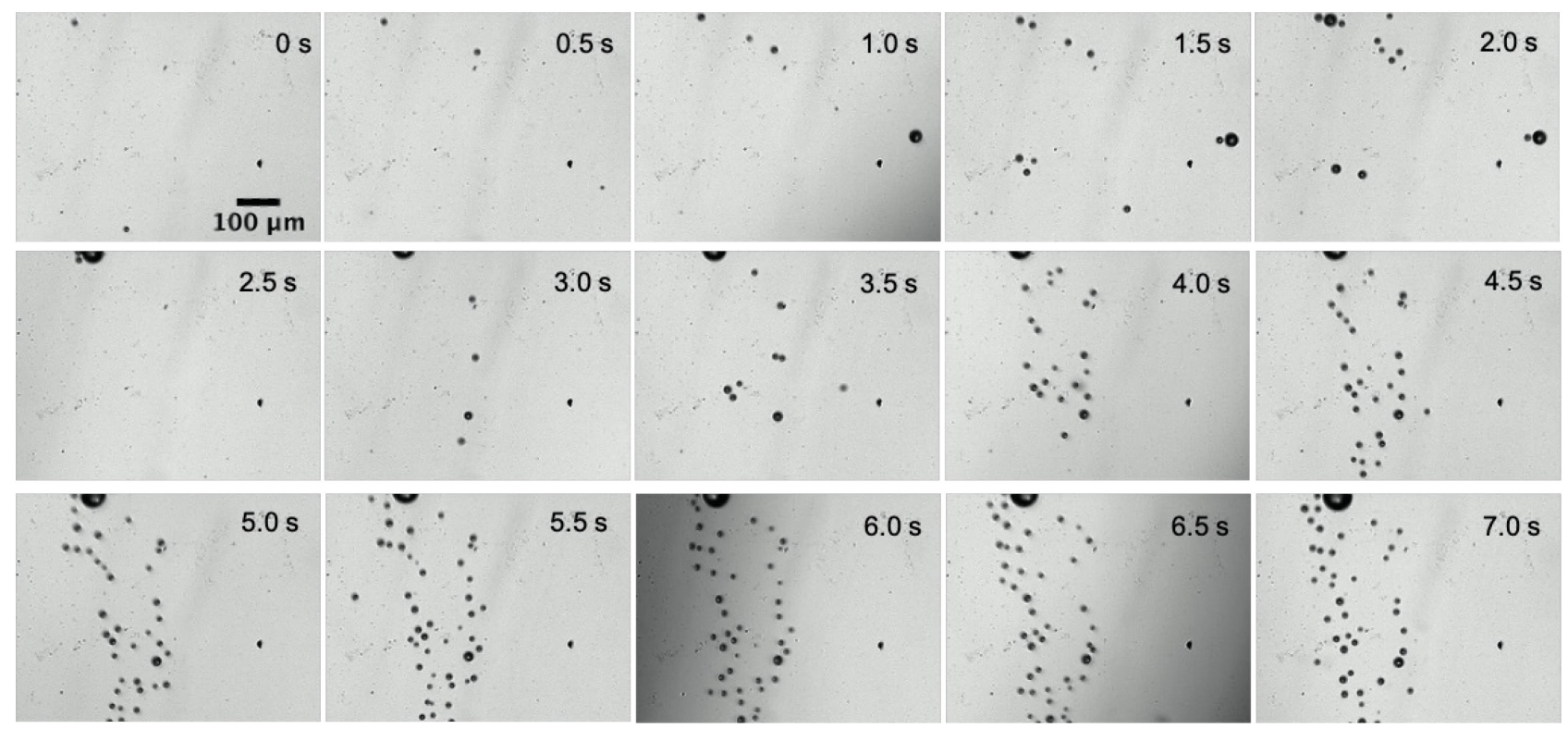

Fig. 3. Close-up visualization (frame rate: $1000 \mathrm{fps}$, exposure time $33.3 \mu \mathrm{s}$, spatial resolution $1.10 \mu \mathrm{m} /$ pixel $)$ of bubbles at different times after the ultrasound irradiation $(20 \mathrm{~W})$ starts.

(a)

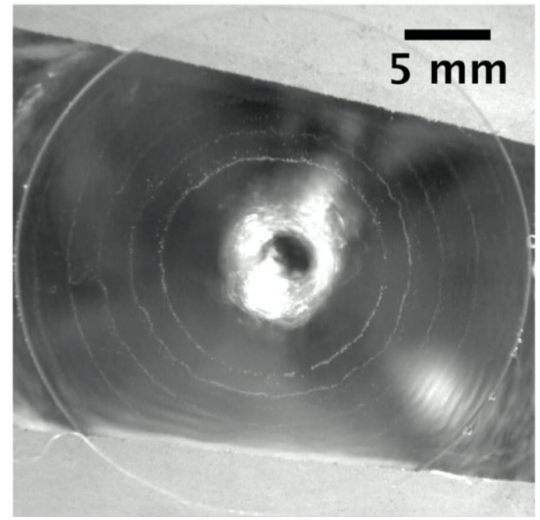

(b)

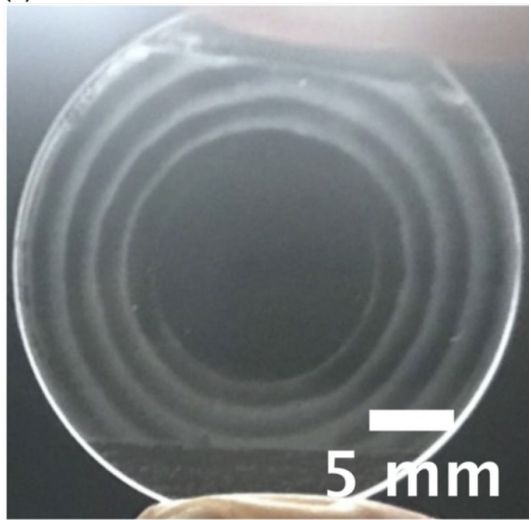

Fig. 4. Visualization of the cleaning test with the higher-intensity $(20 \mathrm{~W})$ ultrasonic water flow. (a) The final frame $(7.0 \mathrm{~s})$ of Fig. 2. (b) Light-scattering patterns on the surface after the 30 -s cleaning. The white region (light schattering due to attached particles) means that particle removal is not achieved. 

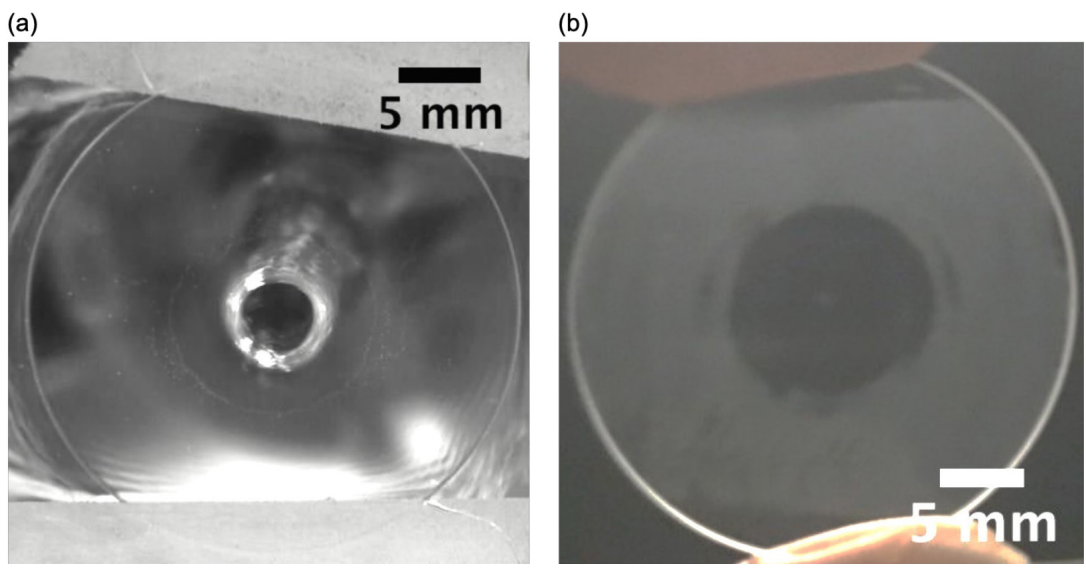

Fig. 5. As figure 4, but with the lower-intensity $(10 \mathrm{~W})$ ultrasonic water flow. (a) Recorded image (frame rate: $30 \mathrm{fps}$, exposure time $16.7 \mathrm{~ms}$, image resolution $62.5 \mu \mathrm{m} / \mathrm{pixel}$ ) of the glass surface (7.0 s after the ultrasound irradiation starts). (b) Light-scattering patterns on the surface after the 30 -s cleaning.

\section{Summary}

The visualization experiment suggests, as expected, that acoustic bubbles play a key role in particle removal in the case of ultrasonic water flow cleaning. It is a technical tip that there is a need to avoid the formation of a standing-wave-like acoustic field in the liquid film flow over the target surface (say, by changing the jet collision location during the cleaning process), which allows for uniform cleaning performance with cavitation bubbles being populated uniformly in space.

\section{References}

[1] T. Kondo and K. Ando: Phys. Fluids 31 (2019), 013303.

[2] W. Kim et al.: Appl. Phys. Lett. 94 (2009), 081908.

[3] T. Yamashita and K. Ando: Ultrason. Sonochem. 52 (2019), 268.

[4] T. Yamashita, R. Yamauchi, and K. Ando: Jpn. J. Multiphase Flow 32 (2018). 210.

[5] T.G. Leighton et. al.: Eur. J. Phys. 11 (1990), 47.

[6] L.A. Crum: J. Acoust. Soc. Am. 68 (1980), 203.

[7] N. Vyas, et al.: Ultrason. Sonochem. 67 (2020), 105112. 